\title{
Pregnancy, Puerperium and Perinatal Condition Class
}

National Cancer Institute

\section{Source}

National Cancer Institute. Pregnancy, Puerperium and Perinatal Condition Class. NCI

Thesaurus. Code C49404.

A class of conditions associated with pregnancy, puerperium and perinatal status. 